\title{
Model a Discourse and Transform It to Your User Interface
}

\author{
Hermann Kaindl \\ Vienna University of Technology \\ Gußhausstr. 27-29, A-1040 Vienna, Austria \\ kaindl@ict.tuwien.ac.at
}

\begin{abstract}
Every interactive system needs a user interface, today possibly even several ones adapted for different devices (PCs, PDAs, mobile phones). Developing a user interface is difficult and takes a lot of effort, since it normally requires design and implementation. This is also expensive, and even more so for several user interfaces for different devices.

This tutorial shows how human-computer interaction can be based on discourse modeling, even without employing speech or natural language. Our discourse models are derived from results of Human Communication theories, Cognitive Science and Sociology. Such discourse models can specify an interaction design. This tutorial also demonstrates how such an interaction design can be used for model-driven generation of user interfaces and linking them to the application logic and the domain of discourse.
\end{abstract}

Keywords: Discourse modeling, model-driven user interface generation.

\section{Tutorial Goals}

The main goals are to show a new approach to modeling discourses, and to demonstrate that and to explain how user interfaces can be generated automatically from high-level discourse models, primarily through transformations using a model-driven approach. Ideally, even end users may be able to model discourses and to transform them into their user interfaces.

\section{Key Learning Outcomes}

In this tutorial, participants learn about modeling discourses using a new approach inspired by human-human communication. They know how modeling discourses and generating user interfaces can be approached systematically. Based on the available tool for automated generation of user interfaces from such models, participants also learn about a new approach to UI prototyping with "perfect fidelity". The result would be identical to the real UI due to this generation process, assuming that the same interaction design according to our approach is taken as input. After a change in the discourse model, the related changes in the UI can be seen more or less immediately. 


\section{References}

1. Bogdan, C., Falb, J., Kaindl, H., Kavaldjian, S., Popp, R., Horacek, H., Arnautovic, E., Szep, A.: Generating an Abstract User Interface from a Discourse Model Inspired by Human Communication. In: Proceedings of the 41st Annual Hawaii International Conference on System Sciences (HICSS 41), Big Island, HI, USA. IEEE Computer Society Press, Los Alamitos (2007)

2. Bogdan, C., Kaindl, H., Falb, J., Popp, R.: Modeling of interaction design by end users through discourse modeling. In: Proceedings of the 2008 ACM International Conference on Intelligent User Interfaces (IUI 2008), Maspalomas, Gran Canaria, Spain. ACM Press, New York (2008)

3. Falb, J., Kaindl, H., Horacek, H., Bogdan, C., Popp, R., Arnautovic, E.: A discourse model for interaction design based on theories of human communication. In: CHI 2006 Extended Abstracts on Human Factors in Computing Systems, pp. 754-759. ACM Press, New York (2006)

4. Falb, J., Kavaldjian, S., Popp, R., Raneburger, D., Arnautovic, E., Kaindl, H.: Fully Automatic User Interface Generation from Discourse Models. In: Proceedings of the 2009 ACM International Conference on Intelligent User Interfaces (IUI 2009), Sanibel Island, Florida, USA. ACM Press, New York (2009); Tool demo paper

5. Falb, J., Popp, R., Röck, T., Jelinek, H., Arnautovic, E., Kaindl, H.: UI Prototyping for Multiple Devices Through Specifying Interaction Design. In: Baranauskas, C., Palanque, P., Abascal, J., Barbosa, S.D.J. (eds.) INTERACT 2007. LNCS, vol. 4662, pp. 136-149. Springer, Heidelberg (2007)

6. Kaindl, H.: A Design Process Based on a Model Combining Scenarios with Goals and Functions. IEEE Transactions on Systems, Man, and Cybernetics (SMC) Part A 30, 537 $551(2000)$

7. Kaindl, H., Jezek, R.: From Usage Scenarios to User Interface Elements in a Few Steps. In: Proceedings of the Fourth International Conference on Computer-Aided Design of User Interfaces (CADUI 2002), Valenciennes, France, May, pp. 91-102. Kluwer Academic Publishers, Dordrecht (2002)

8. Kavaldjian, S., Bogdan, C., Falb, J., Kaindl, H.: Transforming Discourse Models to Structural User Interface Models. In: Giese, H. (ed.) MoDELS 2008. LNCS, vol. 5002, pp. 77 88. Springer, Heidelberg (2008) 